\title{
Comparison of Fecal Microbial Communities between White and Black Pigs
}

\author{
Robin B. Guevarra · Jungman Kim • Son G. Nguyen · Tatsuya Unno*
}

Received: 25 August 2015 / Accepted: September 232015 / Published Online: 31 December 2015

(C) The Korean Society for Applied Biological Chemistry 2015

\begin{abstract}
Meat from black pigs (BP) is in high demand compared with that from modern white pig (WP) breeds such as Landrace pigs owing to its high quality. However, the growth rate of black pigs is slower than that of white pig breeds. We investigated differences in the fecal microbial composition between white and black pigs to explore whether these breeds differed in the composition of their gut microbial communities. The swine gut microbiota was investigated using Illumina's MiSeq-based sequencing technology by targeting the $\mathrm{V} 4$ region of the $16 \mathrm{~S}$ rRNA gene. Our results showed that the composition of the gut microbiota was significantly different between the two pig breeds. While the composition of the WP microbiota shifted according to the growth stage, fewer shifts in composition were observed for the BP gut microbiota. In addition, the WP gut microbiota showed a higher Firmicutes/Bacteroidetes ratio compared with that of BP. A high ratio between these phyla was previously reported as an obesity-linked microbiota composition. Moreover, the WP microbiota contained a significantly higher abundance of cellulolytic bacteria, suggesting a possibility of higher fiber digestion efficiency in WP compared to BP. These findings may be important factors affecting growth performance and energy-harvesting capacities in pigs. Our findings of differences in the gut microbiota composition between the two breeds may provide new leads to understand growth rate variation across pig breeds.
\end{abstract}

Keywords cellulolytic microorganisms $\cdot$ feces $\cdot$ growth rate gut flora $\cdot$ pig breeds

R. B. Guevarra · J. Kim · S. G. Nguyen · T. Unno

Faculty of Biotechnology, College of Applied Life Sciences, SARI, Jeju National University, Jeju 690-756, Republic of Korea

*Corresponding author (T. Unno: tatsu@jejunu.ac.kr)

This is an Open Access article distributed under the terms of the Creative Commons Attribution Non-Commercial License (http://creativecommons. org/licenses/by-nc/3.0/) which permits unrestricted non-commercial use, distribution, and reproduction in any medium, provided the original work is properly cited.

\section{Introduction}

The gut microbiota constitutes one of the most complex mammalian microbial communities and has a major impact on the health status of animals (Fisher, 2012). Sequence analysis of the 16S rRNA gene has been widely used to elucidate the diversity and composition of microbial communities within several animal gut systems, including that of pigs (Ley et al., 2008; Lamendella et al., 2011; Isaacson and Kim, 2012). Recent studies have implicated the gut microbiota as a critical determinant of nutrient uptake, energy regulation, and ultimately, weight and metabolic disorders (Tilg, 2010; DiBaise et al., 2012; Krajmalnik-Brown et al., 2012). Moreover, recent reports of a possible association between the gut microbiota and obesity have placed the focus on the significance of the microbiota for well-being (Ley et al., 2005; Turroni et al., 2008; Tilg, 2010). While large amounts of research have been conducted to date on the human microbiome, only a limited amount of information has been collected about swine gut microbiota (Lamendella et al., 2011).

The breed of the pig is one of the most important sources of variation in the sensory quality of pork (Candek-Potokar et al., 1997), which is also influenced by physiological functions such as growth rate and fat deposition (Wood et al., 2004). For example, white pigs (WP), such as Landrace, are noted for their rapid growth, and their weight at weaning is higher than that of other breeds (Taylor et al., 2005). In contrast, black pigs are known for their slower growth rate and lighter carcass weight when compared with those of the other commercial pig breeds (Hwang et al., 2004a). Moreover, there is a high amount of consumer demand for meat from black pigs (BP) because of their redder meat, higher unsaturated fatty acid content, and higher intramuscular fat content compared with those of commercial white breeds such as Landrace pigs (Jin et al., 2001; Hansen et al., 2006; D.H. et al., 2009). Recently, it has been reported that the pig breed affects the composition of the gut microbiota, and the fecal microbial composition was found to differ between foreign breeds and native pigs (Yang et al., 2014). Although the growth of livestock animals can be affected by a number of factors, including 
genetics, age, antibiotics, and the health status of the animal (Turnbaugh et al., 2006; Looft et al., 2012; Shetty et al., 2013), information about non-genetic factors such as the gut microbiota may also be useful for improving the growth rate of livestock animals.

In the present study, we compared the gut microbiota composition between WP and BP. To date, various studies have reported that feed additives such as organic acids and probiotics could enhance the growth performance of pigs, which is an improvement that has been suggested to be likely mediated through the modulation of the gut microbiota (Zentek et al., 2013; Lahteinen et al., 2014). While BP are favored due to their high-quality meat, their growth rate is lower than other breeds. To our knowledge, the comparison of intestinal microbiota between white and black pigs has not previously been performed, and this is the first study that has investigated the relationship between pig breed and intestinal microbial community by $16 \mathrm{~S}$ rRNA gene sequencing using Illumina's MiSeq technology. The work presented here provides fundamental information on the differences in the fecal microbial composition between white and black pigs, and these differences are discussed with respect to growth rate.

\section{Materials and Methods}

Swine feeding and fecal sampling. A total of six pigs (3 white and 3 black pigs) were used in this study, which were each aged approximately 3 weeks at the beginning of the study. The animals were ear-tagged and were raised in a local pig farm in Jeju Island, Korea. All animals were fed with standard commercial diet based on corn and soybean meal until the end of the experiment. Access to feed and water was given ad libitum. Feeding trials were conducted for 9 weeks. The animals were weighed weekly, and fresh fecal samples were collected from each pig during the early growing stage (3-5 weeks of age) and the late growing stage (1012 weeks of age) after weighing the animals. Fecal samples were collected by rectal swabbing. The samples were immediately transported to the laboratory on ice and stored at $20^{\circ} \mathrm{C}$ until further analysis.

DNA extraction and 16S rRNA gene sequencing. Total DNA was extracted from fecal swabs by using a PowerFecal ${ }^{\circledR}$ DNA Isolation Kit (MO BIO Laboratories, USA). Polymerase chain reaction (PCR) was carried out to amplify the V4 region of the 16S rRNA gene of bacteria and archaea as previously described (Kozich et al., 2013). Two microliters of the total DNA from each sample was used as a template, and amplification was performed in triplicate using a Maxime ${ }^{\mathrm{TM}}$ PCR PreMix Kit (iNtRON Biotechnology, Korea) under the following conditions: $95^{\circ} \mathrm{C}$ for 2 min, followed by 30 cycles of $95^{\circ} \mathrm{C}$ for $20 \mathrm{~s}, 55^{\circ} \mathrm{C}$ for $15 \mathrm{~s}$, and $72^{\circ} \mathrm{C}$ for $1 \mathrm{~min}$, and $72^{\circ} \mathrm{C}$ for $5 \mathrm{~min}$. Obtained PCR products were further gel-purified using an AccuPrep ${ }^{\circledR}$ Gel Purification Kit (Bioneer, Korea). All obtained DNA was quantified using a Qubit ${ }^{\mathbb{R}}$ fluorometer (Thermo Fisher Scientific, USA), and equimolar purified amplicons were pooled prior to sequencing. Amplicons were sequenced using MiSeq platforms (Illumina, USA) at Macrogen Inc. (Korea), according to the manufacturer's instructions.

Sequence processing and analysis. FASTQ files obtained from MiSeq paired end sequencing were deposited at short read archives with the registration number SRP043400. Paired end reads were assembled using PEAR software (Zhang et al., 2014). The resulting FASTQ files were converted to FASTA files, aligned to rRNA sequences in the SILVA database (Quast et al., 2013), screened, and filtered by a Mothur pipeline (Schloss et al., 2009). Artificial erroneous reads were corrected using the pre.cluster Mothur subroutine, and chimeric sequences were removed by using UCHIME (Edgar et al., 2011). Taxonomic classification was performed using Ribosomal Database Project (Cole et al., 2009) training set version 9, followed by the removal of non-archaeal/ bacterial sequences based on the taxonomic classification results. Prior to the cluster analysis, all singleton sequences were removed as suggested previously (Degnan and Ochman, 2012) by using the Mothur split.abund subroutine. To normalize the number of reads per sample, 5,000 sequences were randomly picked from each sample by using the Mothur sub.sample subroutine. Operational taxonomic units (OTUs) were calculated at distance of 0.03 by using the Mothur cluster.split subroutine, and OTU-consensus taxa were determined using the Mothur classify.otu subroutine. Similarity analysis between microbial communities was performed using the Mothur tree.shared subroutine based on the Yue and Clayton theta coefficient (JC and MK, 2005).

Distribution analysis of operational taxonomic units. All OTUs were grouped into two growth stages (weeks 3-6 and weeks 1012) and average abundance was calculated within the growth stages; OTUs where the average abundance was lower than 10 were removed. Cytoscape (Shannon et al., 2003) was used to conduct a network analysis between the two breeds at different growth stages. A circular yFiles layout (yWorks, Germany) was applied to analyze factors influencing the distribution of OTUs. Statistical analysis. Significant differences between microbial communities were examined based on P-values obtained by analysis of molecular variance (AMOVA) and Student's $t$-test. Metastats analysis (Foster, 2003) was also used to conduct differential abundance tests between read-abundance and treatments.

\section{Results and Discussion}

MiSeq-based microbial community analysis. Pyrosequencing has been preferably used for microbial community analysis due to its high accuracy and relatively long read length compared with other next generation sequencing platforms (Gilles et al., 2011; Kim et al., 2013). Recently, a MiSeq-based method for the analysis of microbial communities has been established, which was proven to be capable of sequencing more than 300 samples with a few dozens of dual-indexed primer sets (Teeling and Glockner, 2012; Kim et al., 2013). In this study, a total of $1,151,027$ and 1,113,192 DNA sequences were obtained for WP and BP fecal samples, respectively. After the removal of 


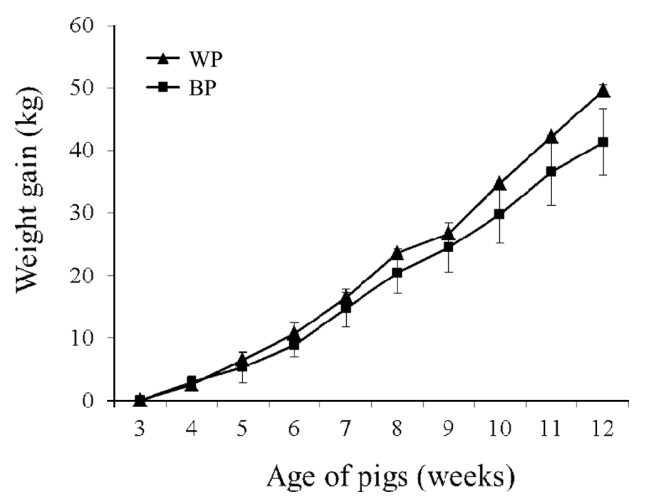

Fig. 1 Growth rate difference between Landrace white pigs (WP) and black pigs (BP).

erroneous reads, 512,957 and 447,814 reads remained for the WP and BP fecal samples, respectively. The number of reads per sample ranged from 7,796 to 51,134 , which was normalized by random-sampling of 5,000 reads by using the Mothur sub.sample subroutine. Results from the rarefaction curve analysis suggest that our sequencing efforts should be enough even after the subsampling (Supplementary Fig. 1).

Growth rate difference between WP and BP. In general, all pigs appeared to be in a good health condition throughout the experiment. WP and BP were fed ad libitum from $18 \pm 2.7$ to $68 \pm 2.9 \mathrm{~kg}$ and from $9.4 \pm 3.5$ to $49.1 \pm 11.4 \mathrm{~kg}$ live weight, respectively. Weekly weight gain was higher in WP and the difference in growth rate between breeds was significant at 12 weeks old (Fig. 1). In Korea, animal breeders have tried to improve BP productivity by crossing them with commercial breeds such as Berkshire and Landrace since 1910 (Kim et al., 2005), which made BP have a close genetic relationship to WP (Kim et al.,
2002; Kim et al., 2005); yet, the growth rate of BP was lower than that of WP. Therefore, non-genetic factors such as the gut microbiota may play a key role in determining growth rate. It has been reported that certain types of gut microbiota could optimize their host's ability to harvest energy from food (Ley et al., 2005; Turnbaugh et al., 2006). In addition, manipulation of gut microbiota was found to control adiposity (Turnbaugh et al., 2006).

Taxonomy-based gut microbiota comparison. A taxonomybased analysis was conducted to describe the composition of the gut microbiome between BP and WP and how it changed over time. The results of phylum distributions are shown in Fig. 2. In WP, the three most abundant phyla, Firmicutes, Bacteroidetes, and Spirochaetes, accounted for approximately $95 \%$ of all obtained phyla, on average. On the other hand, Firmicutes and Bacteroidetes were the two most abundant phyla in BP. A significantly higher relative abundance of Spirochaetes was observed in WP than in BP from 3 to 6 weeks of age $(p<0.05)$. Recently, it has been reported that obese pigs had a higher abundance of the phyla Spirochaetes than did lean pigs (Pedersen et al., 2013). Spirochaetes were originally thought to be nonpathogenic; however, some strains may be enteropathogenic and produce clinical syndromes such as swine dysentery and local inflammation in the colon (; Hampson and Ahmed, 2009). Although it is not a desirable way to increase weight of animals, those gut inflammation in the colon could also contribute to weight gain (de La Serre et al., 2010).

In animal models of obesity, the synergy between the dominant gut phyla is shifted, with a significant reduction of Bacteroidetes and a corresponding increase of Firmicutes (Turnbaugh et al., 2006). In this study, a lower abundance of Bacteroidetes species and a correspondingly higher abundance of Firmicutes species
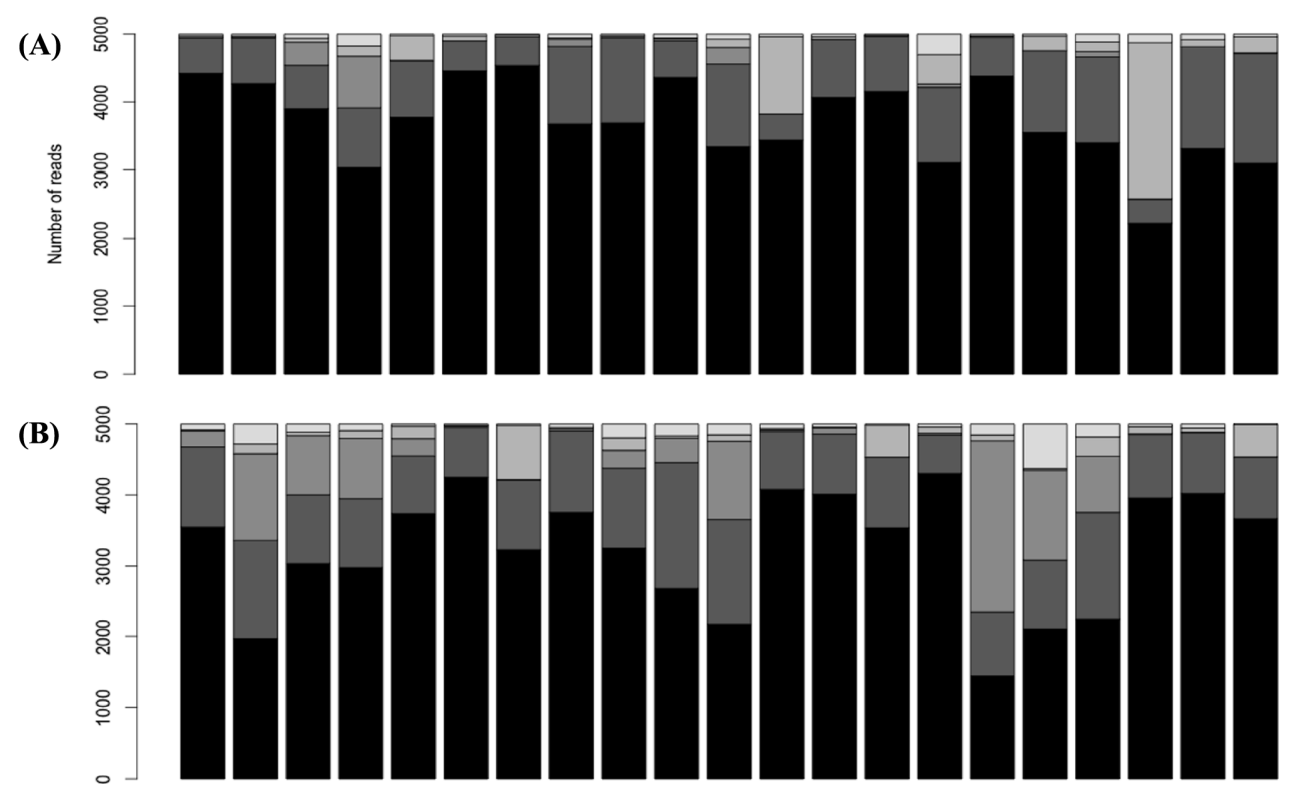

Fig. 2 Phylum-level bacterial composition analysis for white pigs (A) and black pigs (B). W and B denote white and black pigs, respectively. The first number with W and B (W1-3 or B1-3) indicates the individual pigs. Numbers $(3-6,10-12)$ indicates the age of the pigs in weeks. 


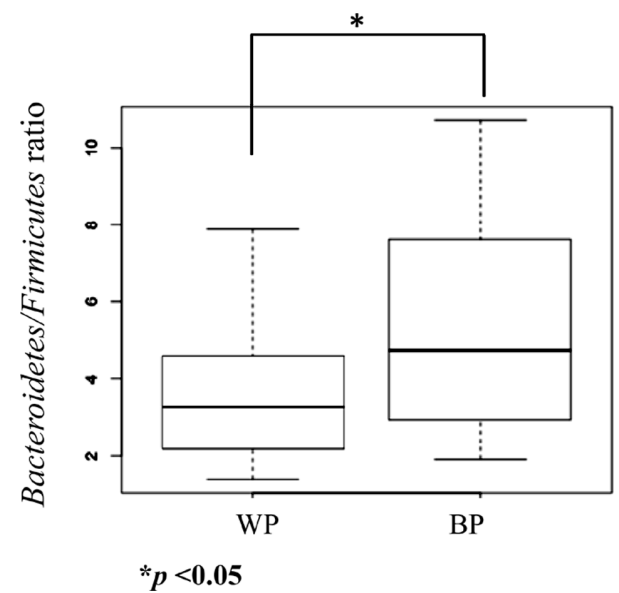

Fig. 3 Bacteroidetes to Firmicutes ratio (B/F ratio) of white (WP) and black pigs (BP).

were observed among WP samples (Fig. 3). A high ratio of Firmicutes to Bacteroidetes abundance has been closely linked with obesity by various studies (Ley et al., 2005; Ley et al., 2006; Turnbaugh et al., 2006; Guo et al., 2008). However, the relationship between the gut microbial composition and the energy-harvesting capacity of the host is complicated and may not be directly related to the Bacteroidetes to Firmicutes ratio (Duncan et al., 2008; Murphy et al., 2010). Further studies are needed to elucidate the mechanisms by which the composition of the gut microbiota may influence fat deposition. Nevertheless, with an increasing amount of evidence supporting the association between alteration of gut microbiota composition and obesity, our results suggest that the ratio of the two phyla might contribute to the lower weight gain of BP compared with WP.

Whole microbiota comparison based on operational taxonomic unit distribution. To verify that BP and WP showed differences in their gut microbial communities, a tree diagram was constructed (Fig. 4). All gut microbiota were divided into two clusters (A and B) and two subclusters (I and II). Cluster A was mostly composed of WP at early stage ( 3 to 6 weeks of age). Cluster B, on the other hand, was divided into two subclusters: subcluster-I contained mostly BP with varying ages and subcluster-II was generally composed of WP at late stage (10 and 11 weeks of age). Analysis by AMOVA suggested that BP and WP have significantly different intestinal microbial communities $(p<0.001)$. Analysis of diversity indices (Supplementary Table 1) showed that WP samples have higher number of OTUs with higher diversity $(p<0.05)$ compared with those of BP. In addition, WP species richness in the early growth stage was significantly higher than that of the late growth stage $(p<0.01)$. Previously, it has been reported that gut microbiota in young swine is still in development, thus difference between individuals could be more noticeable compared to that of adults (Kim et al., 2015). In this study, gut microbiota at late growth stage has been stabilized, which may have minimized bacterial compositional difference between individuals. Our results indicated that WP gut microbiota had higher diversity and species richness

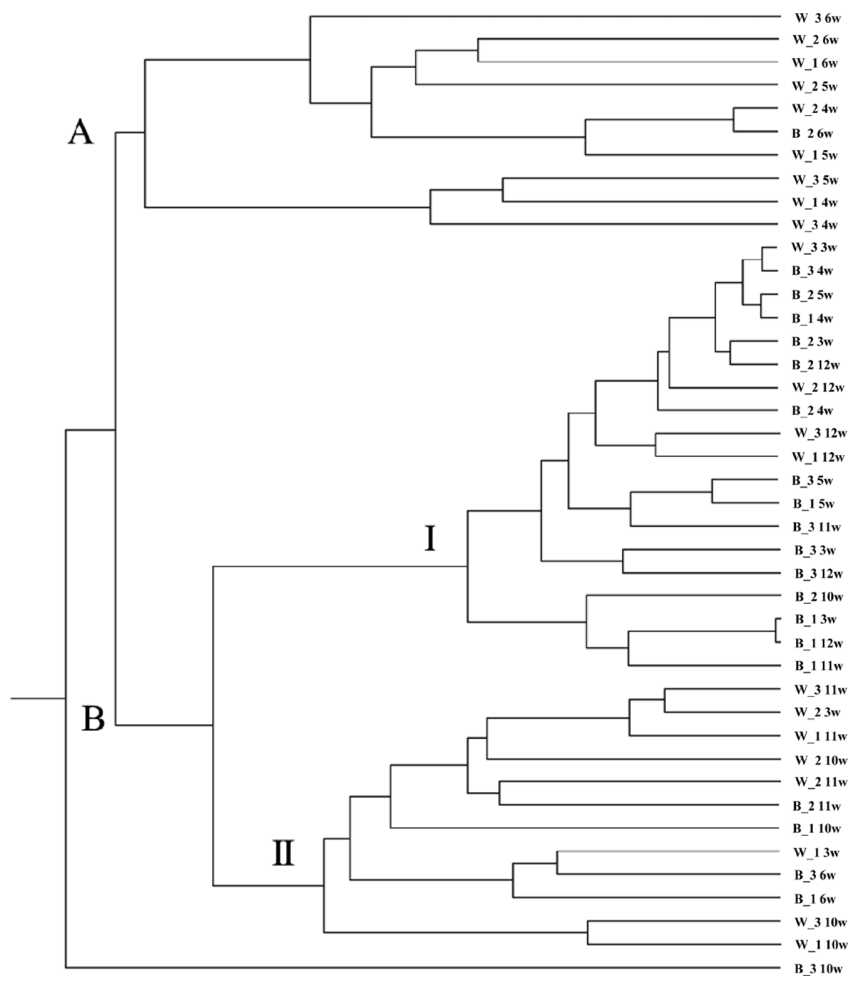

Fig. 4 Cluster analysis of gut microbiota in white and black pigs. Sample names were shown in the following order: pig breed $(\mathrm{W} / \mathrm{B})$, replicate number(1-3), and age of the pigs.

than BP, especially in the early growth stage.

A network analysis was conducted to analyze the distribution of OTUs across breeds, individuals, and growth stages. In Fig. 5, continuous white and grey nodes are observed on the network perimeter. The BP nodes are positioned close to each other and linked with highly abundant OTUs. In contrast, the WP nodes are more frequently distributed on the network perimeter and are separately positioned according to the growth stages. Results from the Fig. 5 suggest that the distribution of OTUs was mostly influenced by breed, and only WP nodes were influenced by growth stage. It has been reported that the swine gut microbiota showed significant shifts after stabilization through the weanling period (Thompson et al., 2008), which is in agreement with our results for WP. On the other hand, the BP nodes were positioned close to the early stage WP nodes, suggesting that the growthstage-dependent shift observed in WP was not observed in BP gut microbiota. Norris et al. (2013) hypothesized that the gut microbiota plays a key role in controlling hosts' appetites, based on previous findings of links between the gut microbiota composition and hosts' behavioral or physiological changes.

Genus level taxonomic classification identified 110 genera in this study. Predominant genera in black pigs were Prevotella, whereas white pigs had Prevotella and Treponema (Supplementary Fig. 2). Since there are quite a few unclassified genera, we conducted OTU-based differential abundance comparison using Metastats analysis (Paulson et al., 2011). Results from the 


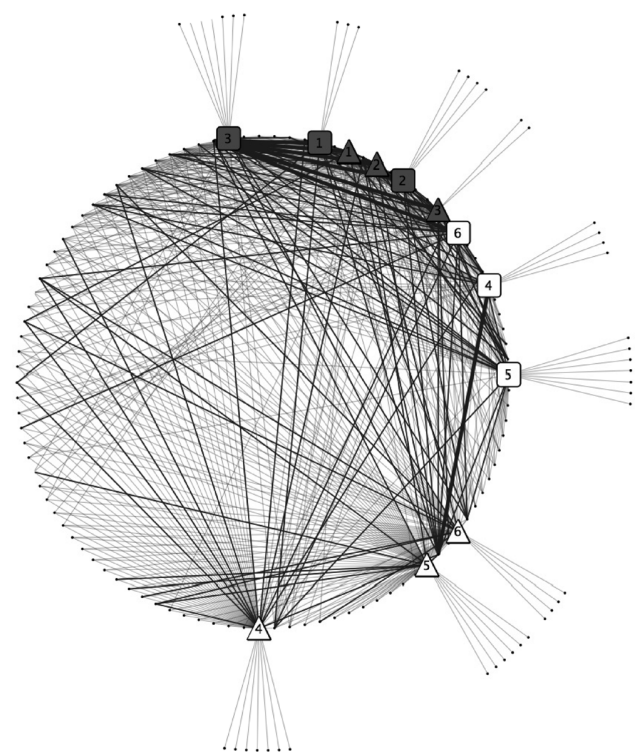

Fig. 5 Network analysis of operational taxonomic units shared between white pigs (WP) and black pigs (BP). Grey and white nodes indicate BP and WP, respectively. Numbers in the nodes indicate the individual pigs. Squares and rectangles indicate early and late growth stages. Line thickness increases according to their read abundance

Metastats analysis (Supplementary Table 2) indicated that WP had significantly higher abundances of the genera Treponema, Cellulosilyticum, Methanobrevibacter, Sporobacter, Anaeroplasma, Peptococcus, Comamonas, and Marinospirillum $(p<0.05$ and $\mathrm{Q}<0.05$ ), while BP had significantly higher abundances of the genera Escherichia, Faecalibacterium, Sutterella, Veillonella, Pasteurella, Mobiluncus, Chlamydia, and Odoribacter $(p<0.05$ and $\mathrm{Q}<0.05$ ). Feeds containing high fiber contents have been increasingly used due to greater availability and lower cost (Ziemer et al., 2012). Since pigs do not have any enzymes to hydrolyze non-starch polysaccharides, their intestinal bacteria play the main role in the digestion and fermentation of those dietary carbohydrates (Choct et al., 2010). In this study, we found two abundant potential fiber digesting helpers in WP, Cellulosilyticum (Cai et al., 2010) and Treponema (Liu et al., 2014). As a result of the bacterial fiber fermentation in colon, short chain fatty acids (SCFA) are produced, which play an important role in maintaining colonic health by supplying energy to colonocytes and protecting them from gastrointestinal disorders (Hijova and Chmelarova, 2007; Cai and Dong, 2010). In addition, energy produced by microflora in the hindgut can satisfy up to $30 \%$ of the maintenance energy requirements of the pig (Adesehinwa, 2008). Moreover, it has been reported that the growth performance of pigs can vary depending on the types of fiber content in feeds (Song et al., 2003). Therefore, fiber digestion also could be one of the key factors determining the growth rate of pigs. In addition, a high abundance of Cellulosilyticum has been reported to positively correlate with the amount of methane production, suggesting that the genus Cellulosilyticum is a key substrate provider for methanogens (Pramanik and Kim, 2014). Our results showed that the genus Methanobrevibacter, a fecal methanogen that utilize acetate, was more prevalent in WP, thus suggesting potentially higher presence of SCFA. Previously, it was suggested that analysis of the fecal methanogen abundance could provide a method to estimate fat storage in pigs, since the abundance of the genus Methanobrevibacter was found to be associated with higher weight gain in pigs (Luo et al., 2012). Existence of methanogens may enhance the activity of polysaccharide-utilizing bacterial species through co-colonization in the gut (Samuel and Gordon, 2006), therefore methanogens could also play an important role in probiotics-mediated energy metabolism and adiposity.

While a number of studies have reported how gut microbiota is associated with obesity (Turnbaugh et al., 2006; Sanz et al., 2008; Tilg, 2010; DiBaise et al., 2012), limited numbers of studies have been reported about gut microbiota shift with respect to livestock animal meat quality. Addition of essential oils increased longissimus muscle while reducing noxious gas in fecal output (Yan et al., 2010), suggesting meat quality could be directly or indirectly associated with gut microbiota. In addition, phenyllactic acid reduced number of $E$. coli while partly ameliorating meat quality (Wang et al., 2010). Moreover, feeding bacteria strain, Clostridium butyricum, improved quality of meat by increasing polyunsaturated fatty acids (Liao et al., 2015). In contrast, it has been reported that the addition of extruded flaxseed improved meat quality without affecting swine gut microbiota (Holman et al., 2014). These studies suggest further study should be conducted to elucidate functional roles of gut microbiota regarding to meat quality.

In summary, we investigated the gut microbiota composition of BP and WP as a potential contributory factor to the differences in the growth rate between the breeds. In this study, feed and water consumption or fecal output of each animal were not recorded. Recently, it has been reported that gut microbiota is also responsible for gut satiety hormone production (Cluny et al., 2015), thus variation in feeding system may provide additional aspects in revealing functional roles of gut microbiota regarding to growth enhancement. To study gut microbiota with respect to feed efficiency, further study should include more controlled feeding system, such as restricted feeding. Although various factors are involved in swine growth performance, our results suggest that gut microbiota difference is one of the factors that affect growth rate in swine. Further study should prove what causes the gut microbiota difference across breeds. In addition, functional genome study by metagenome shotgun sequencing may reveal functional effects resulting from microbiota differences. These information should provide fundamental knowledge toward developing novel approach in enhancing growth performance of livestock animals.

Acknowledgment This work was carried out with the support of the "Cooperative Research Program for Agriculture Science \& Technology Development (Project PJ009782)", Rural Development Administration, Republic of Korea. 


\section{References}

Adesehinwa AOK (2008) Energy and protein requirements of pigs and the utilization of fibrous feedstuffs in Nigeria: A review. Afr $J$ Biotech 7, 4798-806.

Cai S and Dong X (2010) Cellulosilyticum ruminicola gen. nov., sp. nov., isolated from the rumen of yak, and reclassification of Clostridium lentocellum as Cellulosilyticum lentocellum comb. nov. Int J Syst Evol Microbiol 60, 845-9.

Cai S, Li J, Hu FZ, Zhang K, Luo Y, Janto B et al. (2010) Cellulosilyticum ruminicola, a newly described rumen bacterium that possesses redundant fibrolytic-protein-encoding genes and degrades lignocellulose with multiple carbohydrate- borne fibrolytic enzymes. Appl Environ Microbiol 76, 3818-24.

Choct M, Dersjant-Li Y, McLeish J, and Peisker M (2010) Soy Oligosaccharides and Soluble Non-starch Polysaccharides:A Review of Digestion, Nutritive and Anti-nutritive Effects in Pigs and Poultry. AsianAust. Journal of Animal Science 23, 1386-98.

Cluny NL, Eller LK, Keenan CM, Reimer RA, and Sharkey KA (2015) Interactive effects of oligofructose and obesity predisposition on gut hormones and microbiota in diet-induced obese rats. Obesity (Silver Spring) 23, 769-78.

Cole JR, Wang Q, Cardenas E, Fish J, Chai B, Farris RJ et al. (2009) The Ribosomal Database Project: improved alignments and new tools for rRNA analysis. Nucleic Acids Res 37, D141-5.

Degnan PH and Ochman H (2012) Illumina-based analysis of microbial community diversity. ISME J 6, 183-94.

de La Serre CB, Ellis CL, Lee J, Hartman AL, Rutledge JC, and Raybould HE (2010) Propensity to high-fat diet-induced obesity in rats is associated with changes in the gut microbiota and gut inflammation. Am J Physiol Gastrointest Liver Physiol 299, G440-8.

DiBaise JK, Frank DN, and Mathur R (2012) Impact of the Gut Microbiota on the Development of Obesity: Current Concepts. Am J Gastroenterol Suppl 1, 22-7.

Duncan SH, Lobley GE, Holtrop G, Ince J, Johnstone AM, Louis P et al. (2008) Human colonic microbiota associated with diet, obesity and weight loss. Int J Obes (Lond) 32, 1720-4.

Edgar RC, Haas BJ, Clemente JC, Quince C, and Knight R (2011) UCHIME improves sensitivity and speed of chimera detection. Bioinformatics 27, 2194-200.

Fisher AR (2012) Comparison of the gut microbiome of pigs, mice and humans. George Mason University, USA.

Foster EK (2003) METASTATS: behavioral science statistics for Microsoft Windows and the HP49G programmable calculator. Behav Res Methods Instrum Comput 35, 325-8.

Gilles A, Meglecz E, Pech N, Ferreira S, Malausa T, and Martin JF (2011) Accuracy and quality assessment of 454 GS-FLX Titanium pyrosequencing. BMC Genomics 12, 245.

Guo X, Xia X, Tang R, and Wang K (2008) Real-time PCR quantification of the predominant bacterial divisions in the distal gut of Meishan and Landrace pigs. Anaerobe 14, 224-8.

Hampson DJ and Ahmed N (2009) Spirochaetes as intestinal pathogens: Lessons from a Brachyspira genome. Gut Pathog 1, 1-3.

Hijova E and Chmelarova A (2007) Short chain fatty acids and colonic health Bratisl Lek Listy 108, 354-8.

Holman DB, Baurhoo B, and Chenier MR (2014) Temporal analysis of the effect of extruded flaxseed on the swine gut microbiota. Can J Microbio 60, 649-59.

Isaacson R and Kim HB (2012) The intestinal microbiome of the pig. Anim Health Res Rev 13, 100-9.

JC Y and MK C (2005) A similarity measure based on species proportions. Comm Statist Theory Methods 34, 2123-31.

Kim J, Nguyen SG, Guevarra RB, Lee I, and Unno T (2015) Analysis of swine fecal microbiota at various growth stages. Arch Microbiol 197 753-9.

Kim KS, Yeo JS, and Kim JW (2002) Assessment of genetic diversity of Korean native pig (Sus scrofa) using AFLP markers. Genes Genet Syst 77, 361-8.
Kim M, Lee KH, Yoon SW, Kim BS, Chun J, and Yi H (2013) Analytical tools and databases for metagenomics in the next-generation sequencing era. Genomics Inform 11, 102-13.

Kim TH, Kim KS, Choi BH, Yoon DH, Jang GW, Lee KT et al. (2005) Genetic structure of pig breeds from Korea and China using microsatellite loci analysis. J Anim Sci 83, 2255-63.

Kinyon JM and Harris DL (1979) Treponema innocens, a new species of intestinal bacteria, and emended description of the type strain of Treponema hyodysenteriae. Int J Syst Bacteriol 29, 102-9.

Kozich JJ, Westcott SL, Baxter NT, Highlander SK, and Schloss PD (2013) Development of a dual-index sequencing strategy and curation pipeline for analyzing amplicon sequence data on the MiSeq Illumina sequencing platform. Appl Environ Microbiol 79, 5112-20.

Krajmalnik-Brown R, Ilhan ZE, Kang DW, and DiBaise JK (2012) Effects of gut microbes on nutrient absorption and energy regulation. Nutr Clin Pract 27, 201-14.

Lahteinen T, Lindholm A, Rinttila T, Junnikkala S, Kant R, Pietila TE et al. (2014) Effect of Lactobacillus brevis ATCC 8287 as a feeding supplement on the performance and immune function of piglets. Vet Immunol Immunopathol 158, 14-25.

Lamendella R, Domingo JW, Ghosh S, Martinson J, and Oerther DB (2011) Comparative fecal metagenomics unveils unique functional capacity of the swine gut. BMC Microbiol 11, 103.

Ley RE, Backhed F, Turnbaugh P, Lozupone CA, Knight RD, and Gordon JI (2005) Obesity alters gut microbial ecology. Proc Natl Acad Sci U S A 102, 11070-5.

Ley RE, Hamady M, Lozupone C, Turnbaugh PJ, Ramey RR, Bircher JS et al. (2008) Evolution of mammals and their gut microbes. Science $\mathbf{3 2 0}$, $1647-51$.

Ley RE, Turnbaugh PJ, Klein S, and Gordon JI (2006) Microbial ecology: human gut microbes associated with obesity. Nature 444, 1022-3.

Liao X, Wu R, Ma G, Zhao L, Zheng Z, and Zhang R (2015) Effects of Clostridium butyricum on antioxidant properties, meat quality and fatty acid composition of broiler birds. Lipids Health Dis 14, 36.

Liu J, Wang JK, Zhu W, Pu YY, Guan LL, and Liu JX (2014) Monitoring the rumen pectinolytic bacteria Treponema saccharophilum using real-time PCR. FEMS Microbiol Ecol 87, 576-85.

Luo Y, Su Y, Wright AG, Zhang L, Smidt H, and Zhu W (2012) Lean Breed Landrace Pigs Harbor FecalMethanogens at Higher Diversity and Density than Obese Breed Erhualian Pigs. Hindawi Publishing Corporation 1-9.

Murphy EF, Cotter PD, Healy S, Marques TM, O'Sullivan O, Fouhy F et al. (2010) Composition and energy harvesting capacity of the gut microbiota: relationship to diet, obesity and time in mouse models. Gut 59, 1635-42.

Norris V, Molina F, and Gewirtz AT (2013) Hypothesis: bacteria control host appetites. J Bacteriol 195, 411-6.

Paulson J, Pop M, and Bravo H (2011) Metastats: an improved statistical method for analysis of metagenomic data. Genome Biology 12, P17.

Pedersen R, Ingerslev HC, Sturek M, Alloosh M, Cirera S, Christoffersen BO et al. (2013) Characterisation of gut microbiota in Ossabaw and Gottingen minipigs as models of obesity and metabolic syndrome. PloS one 8 , e56612.

Pramanik P and Kim PJ (2014) Evaluating changes in cellulolytic bacterial population to explain methane emissions from air-dried and composted manure treated rice paddy soils. Sci Total Environ 470-471, 1307-12.

Quast C, Pruesse E, Yilmaz P, Gerken J, Schweer T, Yarza P et al. (2013) The SILVA ribosomal RNA gene database project: improved data processing and web-based tools. Nucleic Acids Res 41, D590-6.

Samuel BS and Gordon JI (2006) A humanized gnotobiotic mouse model of host-archaeal-bacterial mutualism. Proc Natl Acad Sci U A 103, 10011-6.

Sanz Y, Santacruz A, and De Palma G (2008) Insights into the roles of gut microbes in obesity. Interdiscip Perspect Infect Dis 2008, 829101.

Schloss PD, Westcott SL, Ryabin T, Hall JR, Hartmann M, Hollister EB et al. (2009) Introducing mothur: open-source, platform-independent, community-supported software for describing and comparing microbial communities. Appl Environ Microbiol 75, 7537-41. 
Shannon P, Markiel A, Ozier O, Baliga NS, Wang JT, Ramage D et al. (2003) Cytoscape: a software environment for integrated models of biomolecular interaction networks. Genome Res 13, 2498-504.

Song GL, Li DF, Piao XS, Chi F, and Yang WJ (2003) Apparent ilea digestibility of amino acids and the digestible and metabolizable energy content of high-oil corn varieties and its effects on growth performance of pigs. Arch Tierernahr 57, 297-306.

Teeling H and Glockner FO (2012) Current opportunities and challenges in microbial metagenome analysis--a bioinformatic perspective. Brief Bioinform 13, 728-42.

Thompson CL, Wang B, and Holmes AJ (2008) The immediate environment during postnatal development has long-term impact on gut community structure in pigs. ISME $J \mathbf{2}, 739-48$.

Tilg H (2010) Obesity, metabolic syndrome, and microbiota: multiple interactions. J Clin Gastroenterol 44 Suppl 1, S16-8.

Turnbaugh PJ, Ley RE, Mahowald MA, Magrini V, Mardis ER, and Gordon JI (2006) An obesity-associated gut microbiome with increased capacity for energy harvest. Nature 444, 1027-31.

Turroni F, Ribbera A, Foroni E, van Sinderen D, and Ventura M (2008) Human gut microbiota and bifidobacteria: from composition to functionality. Antonie Van Leeuwenhoek 94, 35-50.

Wang JP, Lee JH, Yoo JS, Cho JH, Kim HJ, and Kim IH (2010) Effects of phenyllactic acid on growth performance, intestinal microbiota, relative organ weight, blood characteristics, and meat quality of broiler chicks. Poult Sci 89, 1549-55.

Yan L, Wang JP, Kim HJ, Meng QW, Ao X, Hong SM et al. (2010) Influence of essential oil supplementation and diets with different nutrient densities on growth performance, nutrient digestibility, blood characteristics, meat quality and fecal noxious gas content in grower-finisher pigs. Livestock Science 128, 115-22.

Yang L, Bian G, Su Y, and Zhu W (2014) Comparison of faecal microbial community of lantang, bama, erhualian, meishan, xiaomeishan, duroc, landrace, and yorkshire sows. Asian-Australas J Anim Sci 27, 898-906.

Zentek J, Ferrara F, Pieper R, Tedin L, Meyer W, and Vahjen W (2013) Effects of dietary combinations of organic acids and medium chain fatty acids on the gastrointestinal microbial ecology and bacterial metabolites in the digestive tract of weaning piglets. J Anim Sci 91, 3200-10.

Zhang J, Kobert K, Flouri T, and Stamatakis A (2014) PEAR: a fast and accurate Illumina Paired-End reAd mergeR. Bioinformatics 30, 614-20.

Ziemer CJ, Kerr BJ, Weber TE, Arcidiacono S, Morrison M, and Ragauskas A (2012) Effects of feeding fiber-fermenting bacteria to pigs on nutrient digestion, fecal output, and plasma energy metabolites. J Anim Sci 90, 4020-7. 